\title{
The Role of Adjunctive Ascorbic Acid in the Prevention of Colistin-Induced Nephrotoxicity in Critically III Patients: A Retrospective Study
}

\author{
Khalid Al Sulaiman ( $\nabla$ alsulaimankh@hotmail.com ) \\ King Abdulaziz Medical City \\ Ohoud Al Juhani \\ King Abdulaziz University \\ Abdullah Alhammad \\ King Saud University \\ Kholoud Al Aamer \\ King Abdulaziz Medical City

\section{Sara Alshehri} \\ King Abdulaziz Medical City \\ Abdulmohsen Alhuwahmel
}

King Saud bin Abdulaziz University for Health Sciences

Abdullah Kharbosh

Taif University

Areej Alshehri

Ferring Pharmaceuticals

Hanan Al Shareef

University of Tabuk

Ibrahim Al Sulaihim

Central Security Hospitals, Riyadh

Albandari Alghamdi

Princess Nourah bint Abdulrahman University

Shmeylan Al Harbi

King Abdulaziz Medical City

Numan Abdan

King Abdulaziz Medical City

Abdulmalik Al Katheri

King Abdulaziz Medical City

Yousef Alrajhi

King Abdulaziz Medical City

\section{Ramesh Vishwakarma}

European Organization for Research and Treatment of Cancer (EORTC) Headquarters, Brussels

\section{Research Article}

Keywords: Colistin, Ascorbic Acid, Vitamin C, nephrotoxicity, colistin-induced nephrotoxicity, mortality 
DOl: https://doi.org/10.21203/rs.3.rs-1254017/v1

License: (c) (i) This work is licensed under a Creative Commons Attribution 4.0 International License. Read Full License 


\section{Abstract}

\section{Background}

Colistin antibiotic is considered a valuable and last-resort therapeutic option for MDR gram-negative bacteria.

Nephrotoxicity is the most clinically pertinent adverse effect for colistin. Vivo studies suggest that administering oxidative stress-reducing agents, such as ascorbic acid, is a promising strategy to overcome colistin-induced nephrotoxicity (CIN). However, limited clinical data explores the potential benefit of adjunctive ascorbic acid therapy for preventing CIN. Therefore, this study aims to assess the potential nephroprotective role of ascorbic acid as adjunctive therapy against CIN in critically ill patients.

\section{Method}

This was a retrospective cohort study at King Abdulaziz Medical City (KAMC) for all adult critically ill patients who have received IV Colistin. Eligible patients were classified into two groups based on the ascorbic acid use as concomitant therapy within three days of colistin initiation. The primary outcome was CIN odds after colistin initiation, while the secondary outcomes were 30-day mortality, in-hospital mortality, ICU, and hospital LOS. Propensity score (PS) matching was used (1:1 ratio) based on the patient's age, SOFA score, and serum creatinine.

\section{Results}

A total of 451 patients were screened for eligibility; 90 patients were included after propensity score matching based on the selected criteria. The odds of developing CIN after colistin initiation were similar between patients who received ascorbic acid (AA) as adjunctive therapy compared to patients who did not $(\mathrm{OR}(95 \% \mathrm{Cl}): 0.83(0.33,2.10)$, p-value $=0.68)$. In addition, the 30-day mortality, in-hospital mortality, ICU, and hospital LOS were similar between the two groups.

\section{Conclusion}

Concomitant use of Ascorbic acid with colistin was not associated with lower odds of colistin-induced nephrotoxicity. Further studies with a larger sample size are required to confirm these findings.

\section{Introduction}

Nosocomial acquired infections due to multidrug-resistant bacteria (MDR) may be associated with increased mortality risk, especially among critically ill patients, which is considered the leading cause of mortality in intensive care units (ICUs) with a $60 \%$ mortality rate. ${ }^{1}$ Certain factors increase the risk of acquiring severe infections with MDR in the ICU settings, such as invasive interventions, prolonged hospitalization, and prolonged use of broad-spectrum antibiotics. ${ }^{2}$

Recently, the clinical use of Colistin has been increased due to the high rate of bacterial resistance and limited development of new antibiotics with activity against gram-negative bacteria ${ }^{3}$ Colistin (known as Polymyxins $\mathrm{E}$ ) is a bactericidal antibiotic in a concentration-dependent mode against gram-negative bacteria and indicated for life-threatening infections caused by MDR and extensively drug-resistant (XDR) bacteria. ${ }^{3,4}$ It is considered the last-resort antibiotic due to its low therapeutic index and high risk of toxicity. ${ }^{5}$ Despite its reported high toxicity rates, it has relatively high clinical cure rates when given as a monotherapy or combination therapy. Recently, Colistin became a vital option against MDR gram-negative species such as Acinetobacter baumannii, Pseudomonas aeruginosa, and Klebsiella pneumoniae. ${ }^{6}$

Although, the exact mechanism of Colistin-induced nephrotoxicity (CIN) is not well known. ${ }^{5,7}$ However, it may be due to oxidative stress in the epithelium proximal tubules that lead to damage of the mitochondria. ${ }^{5} \mathrm{CIN}$ is developed in approximately $60 \%$ of the patients who received Colistin. 8 Many factors may be associated with the increase the risk of developing $\mathrm{CIN}$, such as underlying comorbidities, concomitant administration of nephrotoxic medications, presence of 
septic shock, administering high doses of Colistin, and different colistin formulations. ${ }^{9}$ Additionally, CIN might be a doselimiting factor; it either prevents administering the optimal therapeutic dose or leads to therapy interruption, increasing the risk of bacterial resistance. ${ }^{10,11}$

There is an evolving assumption about the coadministration of an antioxidant to protect the kidneys. ${ }^{5}$ Ascorbic acid is a water-soluble vitamin classified as a chain-breaking antioxidant and scavenger of free radicals. ${ }^{12}$ Many studies have evaluated the rule of ascorbic acid in critically ill patients, and based on the literature, ascorbic acid did not affect the incidence of acute kidney injury nor prevented the need to start renal replacement therapy. ${ }^{13}$ However, the studies that aimed to assess ascorbic acid rule in critically ill patients have conflicted results.

An early study was conducted on rats to assess the effect of ascorbic acid when co-administered with Colistin found that the coadministration of ascorbic acid resulted in a protective effect against tubular apoptosis and nephrotoxicity induced by Colistin. ${ }^{14}$ However, the clinical data exploring the potential benefit of ascorbic acid adjunctive therapy for preventing CIN is limited. Therefore, this study aims to assess the potential nephroprotective effect of ascorbic acid as an adjunctive therapy against $\mathrm{CIN}$ in critically ill patients.

\section{Methods}

\section{Study design}

This retrospective cohort study included critically ill patients who received intravenous (IV) colistin during ICU stay at King Abdulaziz Medical City (Riyadh). All the patients who met our inclusion criteria during the study period (01/01/2017 $31 / 12 / 2020$ ) were included. All patients were followed until they were discharged from the hospital or died during the inhospital stay, whichever occurred first. The study was approved by Institutional Review Board (IRB) - King Abdullah International Medical Research Center (KAIMRC) in March 2021 (Ref.\#NRC21R.062.02). Informed consent was not required hence it has been waived off from the King Abdullah International Medical Research Center Institutional Review Board, Riyadh, Saudi Arabia due to the retrospective observational nature of the study. All methods were performed in accordance with the relevant guidelines and regulations.

\section{Study participants}

We included adult (age $\geq 18$ years) patients who received IV colistin therapy. Patients were excluded if known to have CKD on HD, received a single dose of colistin, have AKI within 24 hours of ICU admission, no concomitant use of colistin and ascorbic acid for the control group, ICU LOS < 24 hours, or death within 24 hours of ICU admission (Figure 1). Eligible patients were then classified into two groups based on ascorbic acid use as concomitant therapy within three days of colistin initiation.

\section{Study setting}

This study was conducted at King Abdulaziz Medical City, a tertiary-care academic referral hospital in Riyadh, Saudi Arabia. King Abdulaziz Medical city has several ICUs, including adult medical, surgical, trauma, and burn ICUs. The ICUs admit medical, surgical, trauma, burn patients and operate as closed units with $24 / 7$ onsite coverage by critical care boardcertified intensivists and clinical pharmacists.

\section{Data collection}

Each patients' data was collected and retrieved from the hospital system (best care); data was entered in an excel sheet. We collected patients' demographic data, comorbidities, vital signs and severity scores (SOFA and APACHE II), and mechanical ventilation status within 24 hours of ICU admission. Moreover, laboratory tests such as liver and renal profile, complete blood count, and lactic acid were collected. Furthermore, we record the following variables, timing of ascorbic acid and colistin initiation, duration of ascorbic acid and colistin therapy, concomitant use of other nephrotoxic medications (i.e., 
vancomycin, aminoglycosides, and contrast). All patients were followed during ICU stay until they were discharged from the hospital or died during the in-hospital stay.

\section{Outcomes}

This study aims to assess the potential nephroprotective role of ascorbic acid as adjunctive therapy against CIN in critically ill patients. The primary outcome was CIN odds after colistin initiation, while the secondary outcomes were 30-day mortality, in-hospital mortality, ICU and hospital LOS.

\section{Outcome definition (s)}

- The 30-day mortality was defined as a death occurring for any cause within 30 days of the admission date during hospital stay; patients who were discharged from the hospital alive were presumed to be survived.

- Colistin-induced nephrotoxicity (CIN) was defined as a sudden decrease of renal function i.e. within 24 hours after colistin initiation, defined by an increase in absolute serum creatinine $(\mathrm{SCr})$ of at least $26.5 \mu \mathrm{mol} / \mathrm{L}(0.3 \mathrm{mg} / \mathrm{dL})$ or by a percentage increase in $\mathrm{SCr} \geq 50 \%$ (1.5× baseline value) during ICU stay.

\section{Statistical analysis}

As appropriate, we presented numerical variables (continuous variables) as mean with standard deviation (SD), or median with lower and upper quartile (Q1 and Q3), while the categorical variables as number (percentage). The normality assumptions for all numerical variables were evaluated using a statistical test (the Shapiro-Wilk test) and graphical representation (i.e., histograms and Q-Q plots).

The two study groups' baseline characteristics and outcome variables were compared. We used the Chi-square or Fisher's exact test for categorical variables as appropriate. The student t-test was used to compare normally distributed continuous variables and the Mann-Whitney $\mathrm{U}$ test to compare non-normally distributed continuous variables. For the outcomes considered in this study, multivariable logistic, cox proportional hazards, and negative binomial regression analysis were used, and the results were reported as odds ratios (OR), hazard ratios (HR), or estimates with 95\% confidence intervals (Cl) as appropriate. PS score was used as one of the model's covariates in the regression analysis. No imputation was made for missing data as the cohort of patients in our study was not derived from random selection. All statistical analyses were performed using SAS version 9.4, and a P-value of $<0.05$ was considered statistically significant.

Based on patient age, SOFA score, and serum creatinine within 24 hours of ICU admission, the Propensity Score Matching Procedure (Proc PS match) (SAS, Cary, NC) was used to match patients who did not receive ascorbic acid with patients who did receive ascorbic acid as concomitant use with colistin. A greedy nearest neighbor matching method was used, with one patient without ascorbic acid (active) group matched with one patient who received ascorbic acid (control), resulting in the smallest within-pair difference among all available pairs treated patients. The difference in the logits of the propensity scores for pairs of patients from the two groups was matched only if it was less than or equal to 0.5 times the pooled estimate of the standard deviation.

\section{Results}

\section{Demographic and Clinical Characteristics}

Initially, a total of 451 patients were screened for eligibility, of which 276 patients were included. Patients who administered colistin-only were 231 patients with a mean age of 54.4 years (SD \pm 20.50$)$, compared to patients who administered colistin concomitant with ascorbic acid therapy (mean age: 47.2 years ( $S D \pm 16.80$ ). The majority of patients included in both groups were male (69.9\%). The most prevalent underlying comorbidities in both groups were diabetes mellitus (41.7\%), followed by hypertension (40.9\%), dyslipidemia (13\%), and stroke (12.7\%) (Table 1). 
Table 1

Baseline characteristics

\begin{tabular}{|c|c|c|c|c|c|c|c|c|}
\hline & \multicolumn{4}{|c|}{ Before propensity score (PS) matching } & \multicolumn{4}{|c|}{ After propensity score (PS) matching } \\
\hline & $\begin{array}{l}\text { Overall } \\
(\mathrm{N}=276)\end{array}$ & $\begin{array}{l}\text { Colistin } \\
(\mathrm{N}=231)\end{array}$ & $\begin{array}{l}\text { Colistin + } \\
\text { Ascorbic } \\
\text { Acid }\end{array}$ & P-value & $\begin{array}{l}\text { Overall } \\
(\mathrm{N}=90)\end{array}$ & $\begin{array}{l}\text { Colistin } \\
(\mathrm{N}=45)\end{array}$ & $\begin{array}{l}\text { Colistin + } \\
\text { Ascorbic } \\
\text { Acid }\end{array}$ & P-value \\
\hline & & & $(\mathrm{N}=45)$ & & & & $(\mathrm{N}=45)$ & \\
\hline $\begin{array}{l}\text { Age (Years), Mean } \\
\text { (SD) }\end{array}$ & $\begin{array}{l}53.2 \\
(20.09)\end{array}$ & $\begin{array}{l}54.4 \\
(20.50)\end{array}$ & $\begin{array}{l}47.2 \\
(16.80)\end{array}$ & $0.0299^{\wedge}$ & $\begin{array}{l}45.5 \\
(17.74)\end{array}$ & $\begin{array}{l}43.8 \\
(18.68)\end{array}$ & $\begin{array}{l}47.2 \\
(16.80)\end{array}$ & $0.2811^{\wedge}$ \\
\hline $\begin{array}{l}\text { Gender - Male, } n \\
\text { (\%) }\end{array}$ & $\begin{array}{l}193 \\
69.9\end{array}$ & $\begin{array}{l}161 \\
69.7\end{array}$ & $32(71.1$ & $0.8499^{\wedge \wedge}$ & )$^{67(74.4}$ & $\begin{array}{l}35( \\
77.8)\end{array}$ & $32(71.1$ & $0.4684^{\wedge \wedge}$ \\
\hline $\begin{array}{l}\text { Weight (kg), Mean } \\
\text { (SD) }\end{array}$ & $\begin{array}{l}76.1 \\
(20.90)\end{array}$ & $\begin{array}{l}75.1 \\
(20.95)\end{array}$ & $\begin{array}{l}80.9 \\
(20.19)\end{array}$ & $0.0875^{\wedge}$ & $\begin{array}{l}75.6 \\
(22.29)\end{array}$ & $\begin{array}{l}70.3 \\
(23.24)\end{array}$ & $\begin{array}{l}80.9 \\
(20.19)\end{array}$ & $0.0230 *$ \\
\hline BMI, Mean (SD) & $\begin{array}{l}28.4 \\
(7.79)\end{array}$ & $\begin{array}{l}28.2 \\
(7.61)\end{array}$ & $\begin{array}{l}29.8 \\
(8.63)\end{array}$ & $0.3817^{\wedge}$ & $\begin{array}{l}27.7 \\
(8.18)\end{array}$ & $\begin{array}{l}25.7 \\
(7.25)\end{array}$ & $\begin{array}{l}29.8 \\
(8.63)\end{array}$ & $0.0388^{\wedge}$ \\
\hline $\begin{array}{l}\text { APACHE II score, } \\
\text { Median (Q1, Q3) }\end{array}$ & $\begin{array}{l}17.0 \\
(12.00 \\
26.00)\end{array}$ & $\begin{array}{l}18.0 \\
(12.00 \\
27.00)\end{array}$ & $\begin{array}{l}12.0 \\
(11.00 \\
23.50)\end{array}$ & $0.0869^{\wedge}$ & $\begin{array}{l}14.0 \\
(10.00 \\
24.00)\end{array}$ & $\begin{array}{l}15.0 \\
(10.00 \\
24.00)\end{array}$ & $\begin{array}{l}12.0 \\
(11.00 \\
23.50)\end{array}$ & $0.9251^{\wedge}$ \\
\hline $\begin{array}{l}\text { SOFA score, } \\
\text { Median (Q1, Q3) }\end{array}$ & $\begin{array}{l}7.0(5.00 \\
9.00)\end{array}$ & $\begin{array}{l}7.0 \\
(5.00 \\
9.00)\end{array}$ & $\begin{array}{l}6.0(4.00 \\
7.00)\end{array}$ & $0.0096^{\wedge}$ & $\begin{array}{l}6.0(4.00 \\
7.00)\end{array}$ & $\begin{array}{l}6.0 \\
(4.00 \\
7.00)\end{array}$ & $\begin{array}{l}6.0(4.00 \\
7.00)\end{array}$ & $0.5523^{*}$ \\
\hline $\begin{array}{l}\text { Mechanical } \\
\text { Ventilation, } \mathrm{n}(\%)\end{array}$ & $\begin{array}{l}214 \\
77.5\end{array}$ & $\begin{array}{l}188 \\
81.4\end{array}$ & $26(57.8$ & $0.0005^{\star \star}$ & $3(3.3)$ & $1(2.2)$ & $2(4.4)$ & $0.2004 \star \star$ \\
\hline $\begin{array}{l}\text { PaO2/FiO2 ratio, } \\
\text { Median (Q1, Q3) }\end{array}$ & $\begin{array}{l}199.8 \\
(132.50 \\
324.00)\end{array}$ & $\begin{array}{l}199.0 \\
(132.50 \\
324.00)\end{array}$ & $\begin{array}{l}233.0 \\
(137.90 \\
341.40)\end{array}$ & $0.4252^{\wedge}$ & $\begin{array}{l}232.8 \\
(135.30 \\
376.10)\end{array}$ & $\begin{array}{l}223.9 \\
(134.85, \\
383.60)\end{array}$ & $\begin{array}{l}233.0 \\
(137.90 \\
341.40)\end{array}$ & $0.7766^{\wedge}$ \\
\hline $\begin{array}{l}\text { ALT, Median (Q1, } \\
\text { Q3) }\end{array}$ & $\begin{array}{l}33.0 \\
(19.00 \\
63.00)\end{array}$ & $\begin{array}{l}33.0 \\
(18.00 \\
68.00)\end{array}$ & $\begin{array}{l}33.0 \\
(21.00 \\
56.00)\end{array}$ & $0.7478^{\wedge}$ & $\begin{array}{l}30.5 \\
(18.00 \\
56.00)\end{array}$ & $\begin{array}{l}28.0 \\
(17.00 \\
54.00)\end{array}$ & $\begin{array}{l}33.0 \\
(21.00 \\
56.00)\end{array}$ & $0.5914^{\wedge}$ \\
\hline $\begin{array}{l}\text { AST, Median (Q1, } \\
\text { Q3) }\end{array}$ & $\begin{array}{l}41.0 \\
(25.00 \\
70.00)\end{array}$ & $\begin{array}{l}42.0 \\
(25.00 \\
72.00)\end{array}$ & $\begin{array}{l}37.0 \\
(25.00 \\
55.00)\end{array}$ & $0.2809^{\wedge}$ & $\begin{array}{l}36.5 \\
(22.00 \\
56.00)\end{array}$ & $\begin{array}{l}35.0 \\
(21.00 \\
66.00)\end{array}$ & $\begin{array}{l}37.0 \\
(25.00 \\
55.00)\end{array}$ & $0.7775^{\wedge}$ \\
\hline $\begin{array}{l}\text { Estimated } \\
\text { glomerular } \\
\text { filtration rate } \\
\text { (eGFR) Baseline, } \\
\text { Median }(Q 1, Q 3)\end{array}$ & $\begin{array}{l}96.0 \\
(70.00 \\
130.00)\end{array}$ & $\begin{array}{l}96.0 \\
(70.00 \\
134.00)\end{array}$ & $\begin{array}{l}93.0 \\
(67.00 \\
124.00)\end{array}$ & $0.6034^{\wedge}$ & $\begin{array}{l}101.0 \\
(65.00 \\
141.00)\end{array}$ & $\begin{array}{l}109.0 \\
(61.00 \\
154.00)\end{array}$ & $\begin{array}{l}93.0 \\
(67.00 \\
124.00)\end{array}$ & $0.1927 *$ \\
\hline $\begin{array}{l}\text { Serum creatinine } \\
\text { (mmol/l), Median } \\
(\mathrm{Q} 1, \mathrm{Q})\end{array}$ & $\begin{array}{l}72.5 \\
(58.00 \\
103.50)\end{array}$ & $\begin{array}{l}72.0 \\
(57.00 \\
101.00)\end{array}$ & $\begin{array}{l}81.0 \\
(62.00 \\
112.00)\end{array}$ & $0.2388^{\wedge}$ & $\begin{array}{l}75.0 \\
(58.00 \\
108.00)\end{array}$ & $\begin{array}{l}73.0 \\
(57.00 \\
101.00)\end{array}$ & $\begin{array}{l}81.0 \\
(62.00 \\
112.00)\end{array}$ & $0.3682^{\wedge}$ \\
\hline $\begin{array}{l}\text { UOP (mls/kg/hour), } \\
\text { Mean (SD) }\end{array}$ & $\begin{array}{l}1.1 \\
(0.89)\end{array}$ & $\begin{array}{l}1.1 \\
(0.93)\end{array}$ & $\begin{array}{l}1.1 \\
(0.61)\end{array}$ & $0.4253^{\wedge}$ & $\begin{array}{l}0.9(0.57 \\
1.55)\end{array}$ & $\begin{array}{l}1.0 \\
(0.53 \\
1.61)\end{array}$ & $\begin{array}{l}0.9(0.63 \\
1.53)\end{array}$ & $0.8941^{\wedge}$ \\
\hline $\begin{array}{l}\text { BUN, Median } \\
(Q 1, Q 3)\end{array}$ & $\begin{array}{l}6.9(4.70 \\
11.75)\end{array}$ & $\begin{array}{l}7.4 \\
(5.00 \\
12.20)\end{array}$ & $\begin{array}{l}5.0(4.00 \\
6.90)\end{array}$ & $0.0006^{\wedge}$ & $\begin{array}{l}5.6(4.00 \\
9.90)\end{array}$ & $\begin{array}{l}6.4 \\
(4.50 \\
11.90)\end{array}$ & $\begin{array}{l}5.0(4.00 \\
6.90)\end{array}$ & $0.0719^{\wedge}$ \\
\hline $\begin{array}{l}\text { Bicarbonate, } \\
\text { Median (Q1, Q3) }\end{array}$ & $\begin{array}{l}22.0 \\
(19.00 \\
26.00)\end{array}$ & $\begin{array}{l}22.5 \\
(19.00 \\
26.00)\end{array}$ & $\begin{array}{l}21.0 \\
(18.00 \\
25.00)\end{array}$ & $0.0625^{\wedge}$ & $\begin{array}{l}21.0 \\
(19.00 \\
24.33)\end{array}$ & $\begin{array}{l}22.0 \\
(20.00 \\
24.00)\end{array}$ & $\begin{array}{l}21.0 \\
(18.00 \\
25.00)\end{array}$ & $0.3691^{\wedge}$ \\
\hline
\end{tabular}




\begin{tabular}{|c|c|c|c|c|c|c|c|c|}
\hline \multirow[b]{2}{*}{$\begin{array}{l}\text { Chloride (CL), } \\
\text { Median (Q1, Q3) }\end{array}$} & \multicolumn{4}{|c|}{ Before propensity score (PS) matching } & \multicolumn{4}{|c|}{ After propensity score (PS) matching } \\
\hline & $\begin{array}{l}106.0 \\
(102.00 \\
112.00)\end{array}$ & $\begin{array}{l}106.0 \\
(101.00 \\
112.00)\end{array}$ & $\begin{array}{l}106.0 \\
(103.00 \\
110.00)\end{array}$ & $0.6174^{\wedge}$ & $\begin{array}{l}106.0 \\
(103.00 \\
112.00)\end{array}$ & $\begin{array}{l}108.0 \\
(104.00 \\
113.00)\end{array}$ & $\begin{array}{l}106.0 \\
(103.00 \\
110.00)\end{array}$ & $0.1316^{*}$ \\
\hline $\begin{array}{l}\text { Potassium, Median } \\
(\text { Q1, Q3) }\end{array}$ & $\begin{array}{l}4.1(3.70 \\
4.60)\end{array}$ & $\begin{array}{l}4.1 \\
(3.70 \\
4.60)\end{array}$ & $\begin{array}{l}4.2(3.90 \\
4.70)\end{array}$ & $0.0890^{\wedge}$ & $\begin{array}{l}4.2(3.80 \\
4.60)\end{array}$ & $\begin{array}{l}4.0 \\
(3.70 \\
4.50)\end{array}$ & $\begin{array}{l}4.2(3.90 \\
4.70)\end{array}$ & $0.1087^{\wedge}$ \\
\hline $\begin{array}{l}\text { Sodium, Median } \\
(\mathrm{Q} 1, \mathrm{Q} 3)\end{array}$ & $\begin{array}{l}139.0 \\
(136.00 \\
144.00)\end{array}$ & $\begin{array}{l}140.0 \\
(136.00 \\
144.70)\end{array}$ & $\begin{array}{l}138.0 \\
(136.00 \\
141.00)\end{array}$ & $0.0834^{\wedge}$ & $\begin{array}{l}139.0 \\
(136.00 \\
143.00)\end{array}$ & $\begin{array}{l}140.0 \\
(137.00 \\
143.00)\end{array}$ & $\begin{array}{l}138.0 \\
(136.00 \\
141.00)\end{array}$ & $0.0564^{\star}$ \\
\hline $\begin{array}{l}\text { Platelets count } \\
\text { Baseline }\left(10^{\wedge} 9 / \mathrm{L}\right) \text {, } \\
\text { Median }(\mathrm{Q} 1, \mathrm{Q} 3)\end{array}$ & $\begin{array}{l}250.0 \\
(156.00 \\
337.00)\end{array}$ & $\begin{array}{l}240.0 \\
(149.00 \\
337.00)\end{array}$ & $\begin{array}{l}268.0 \\
(207.00 \\
335.00)\end{array}$ & $0.0851^{\wedge}$ & $\begin{array}{l}262.5 \\
(167.00 \\
343.00)\end{array}$ & $\begin{array}{l}241.0 \\
(154.00 \\
346.00)\end{array}$ & $\begin{array}{l}268.0 \\
(207.00 \\
335.00)\end{array}$ & $0.1627^{\wedge}$ \\
\hline $\begin{array}{l}\text { Lactic acid, Median } \\
(\text { Q1, Q3) }\end{array}$ & $\begin{array}{l}1.6(1.10 \\
2.60)\end{array}$ & $\begin{array}{l}1.6 \\
(1.09 \\
2.48)\end{array}$ & $\begin{array}{l}1.7(1.30 \\
2.80)\end{array}$ & $0.2962^{\wedge}$ & $\begin{array}{l}1.5(1.10 \\
2.60)\end{array}$ & $\begin{array}{l}1.5 \\
(0.91 \\
2.18)\end{array}$ & $\begin{array}{l}1.7(1.30 \\
2.80)\end{array}$ & $0.0624^{\wedge}$ \\
\hline $\begin{array}{l}\text { Total WBC Baseline } \\
\left(10^{\wedge} 9 / \mathrm{L}\right), \text { Median } \\
\left(Q_{1}, Q 3\right)\end{array}$ & $\begin{array}{l}11.7 \\
(8.20 \\
15.40)\end{array}$ & $\begin{array}{l}12.0 \\
(8.50 \\
15.40)\end{array}$ & $\begin{array}{l}11.1 \\
(7.00 \\
15.40)\end{array}$ & $0.3404^{\wedge}$ & $\begin{array}{l}11.1 \\
(7.40 \\
14.20)\end{array}$ & $\begin{array}{l}11.0 \\
(7.60 \\
14.00)\end{array}$ & $\begin{array}{l}11.1 \\
(7.00 \\
15.40)\end{array}$ & $0.9036^{\wedge}$ \\
\hline $\begin{array}{l}\text { International } \\
\text { normalized ratio } \\
\text { (INR), Median } \\
(\text { Q1,Q3) }\end{array}$ & $\begin{array}{l}1.1(1.07 \\
1.27)\end{array}$ & $\begin{array}{l}1.2 \\
(1.08 \\
1.28)\end{array}$ & $\begin{array}{l}1.1(1.00 \\
1.25)\end{array}$ & $0.0318^{\wedge}$ & $\begin{array}{l}1.1(1.00 \\
1.25)\end{array}$ & $\begin{array}{l}1.1 \\
(1.08 \\
1.25)\end{array}$ & $\begin{array}{l}1.1(1.00 \\
1.25)\end{array}$ & $0.2838^{\wedge}$ \\
\hline $\begin{array}{l}\text { Activated partial } \\
\text { thromboplastin } \\
\text { time (aPTT) } \\
\text { Baseline } \\
\text { (Seconds), Median } \\
\text { (Q1, Q3) }\end{array}$ & $\begin{array}{l}28.8 \\
(25.90 \\
33.00)\end{array}$ & $\begin{array}{l}28.9 \\
(26.00 \\
33.00)\end{array}$ & $\begin{array}{l}27.6 \\
(25.10 \\
32.00)\end{array}$ & $0.1803^{\wedge}$ & $\begin{array}{l}28.3 \\
(25.50 \\
32.00)\end{array}$ & $\begin{array}{l}29.0 \\
(26.10 \\
31.75)\end{array}$ & $\begin{array}{l}27.6 \\
(25.10 \\
32.00)\end{array}$ & $0.4236^{\wedge}$ \\
\hline $\begin{array}{l}\text { Total bilirubin } \\
\text { ( }(\text { mol/L), Median } \\
(\mathrm{Q} 1, \mathrm{Q} 3)\end{array}$ & $\begin{array}{l}14.4 \\
(8.30 \\
23.70)\end{array}$ & $\begin{array}{l}14.6 \\
(8.40 \\
25.90)\end{array}$ & $\begin{array}{l}13.2 \\
(7.80 \\
20.00)\end{array}$ & $0.1688^{\wedge}$ & $\begin{array}{l}13.8 \\
(8.00 \\
21.60)\end{array}$ & $\begin{array}{l}14.4 \\
(9.60 \\
23.00)\end{array}$ & $\begin{array}{l}13.2 \\
(7.80 \\
20.00)\end{array}$ & $0.2867^{\wedge}$ \\
\hline $\begin{array}{l}\text { Albumin Baseline } \\
\text { (gm/L), Median } \\
(\mathrm{Q} 1, \mathrm{Q} 3)\end{array}$ & $\begin{array}{l}30.0 \\
(27.00 \\
34.00)\end{array}$ & $\begin{array}{l}30.0 \\
(27.00 \\
34.00)\end{array}$ & $\begin{array}{l}32.0 \\
(28.00 \\
36.00)\end{array}$ & $0.0845^{\wedge}$ & $\begin{array}{l}32.0 \\
(28.00 \\
36.00)\end{array}$ & $\begin{array}{l}32.0 \\
(28.00 \\
36.00)\end{array}$ & $\begin{array}{l}32.0 \\
(28.00 \\
36.00)\end{array}$ & $0.6391^{*}$ \\
\hline $\begin{array}{l}\text { Blood sugar } \\
\text { level (mmol/L) } \\
\text { Baseline, Median } \\
(\mathrm{Q} 1, \mathrm{Q} 3)\end{array}$ & $\begin{array}{l}8.7(6.30 \\
12.70)\end{array}$ & $\begin{array}{l}8.7 \\
(6.30 \\
12.70)\end{array}$ & $\begin{array}{l}8.7(6.70 \\
12.60)\end{array}$ & $0.9306^{\wedge}$ & $\begin{array}{l}8.2(6.30 \\
12.30)\end{array}$ & $\begin{array}{l}8.1 \\
(6.10 \\
11.60)\end{array}$ & $\begin{array}{l}8.7(6.70 \\
12.60)\end{array}$ & $0.5584^{\wedge}$ \\
\hline $\begin{array}{l}\text { GCS Score, Median } \\
(\text { Q1, Q3) }\end{array}$ & $\begin{array}{l}7.0(3.00 \\
13.00)\end{array}$ & $\begin{array}{l}7.0 \\
(3.00 \\
11.00)\end{array}$ & $\begin{array}{l}9.0(3.00 \\
15.00)\end{array}$ & $0.3100^{\wedge}$ & $\begin{array}{l}9.0(3.00 \\
15.00)\end{array}$ & $\begin{array}{l}8.5 \\
(3.50 \\
15.00)\end{array}$ & $\begin{array}{l}9.0(3.00 \\
15.00)\end{array}$ & $0.9530^{\wedge}$ \\
\hline $\begin{array}{l}\text { Vasoactive } \\
\text { Inotropic Score } \\
\text { (VIS), Mean (SD) }\end{array}$ & $\begin{array}{l}31.2 \\
(222.61)\end{array}$ & $\begin{array}{l}34.0 \\
(241.11)\end{array}$ & $\begin{array}{l}16.5 \\
(76.11)\end{array}$ & $0.1903^{\wedge}$ & $\begin{array}{l}10.3 \\
(54.33)\end{array}$ & $\begin{array}{l}4.0 \\
(9.88)\end{array}$ & $\begin{array}{l}16.5 \\
(76.11)\end{array}$ & $0.9052^{\wedge}$ \\
\hline $\begin{array}{l}\text { Ascorbic Acid Dose } \\
\text { (mg/day) Median } \\
(\mathrm{Q} 1, \text { Q3) }\end{array}$ & $\begin{array}{l}1000.0 \\
(1000.00 \\
1000.00)\end{array}$ & NA & $\begin{array}{l}1000.0 \\
(1000.00 \\
1000.00)\end{array}$ & NA & $\begin{array}{l}1000.0 \\
(1000.00 \\
1000.00)\end{array}$ & NA & $\begin{array}{l}1000.0 \\
(1000.00 \\
1000.00)\end{array}$ & NA \\
\hline $\begin{array}{l}\text { Duration of } \\
\text { Ascorbic Acid } \\
\text { (days) Median (Q1, } \\
\text { Q3) }\end{array}$ & $\begin{array}{l}36.0 \\
(15.00 \\
69.00)\end{array}$ & NA & $\begin{array}{l}36.0 \\
(15.00 \\
69.00)\end{array}$ & NA & $\begin{array}{l}36.0 \\
(15.00 \\
69.00)\end{array}$ & NA & $\begin{array}{l}36.0 \\
(15.00 \\
69.00)\end{array}$ & NA \\
\hline
\end{tabular}


Before propensity score (PS) matching

\begin{tabular}{|c|c|c|c|c|c|c|c|c|}
\hline $\begin{array}{l}\text { Duration of Colistin } \\
\text { (days) Median (Q1, } \\
\text { Q3) }\end{array}$ & $\begin{array}{l}10.0 \\
(4.00 \\
19.00)\end{array}$ & $\begin{array}{l}10.0 \\
(4.00 \\
19.00)\end{array}$ & $\begin{array}{l}14.0 \\
(5.00 \\
21.50)\end{array}$ & $0.1525^{\wedge}$ & $\begin{array}{l}11.0 \\
(5.00 \\
21.00)\end{array}$ & $\begin{array}{l}11.0 \\
(4.00 \\
21.00)\end{array}$ & $\begin{array}{l}14.0 \\
(5.00 \\
21.50)\end{array}$ & $0.5718^{\wedge}$ \\
\hline $\begin{array}{l}\text { Concomitant use } \\
\text { of Vancomycin use } \\
\text { for } 2 \text { days or more, } \\
n(\%)\end{array}$ & $\begin{array}{l}208 \\
75.4\end{array}$ & $\begin{array}{l}172 \\
74.5\end{array}$ & )$^{36(80.0}$ & $0.4300^{\wedge \wedge}$ & $75(83.3$ & $\begin{array}{l}39( \\
86.7)\end{array}$ & )$^{36(80.0}$ & $0.3961^{\wedge \wedge}$ \\
\hline $\begin{array}{l}\text { Contrast use during } \\
\text { ICU, n (\%) }\end{array}$ & $\begin{array}{l}165( \\
59.8\end{array}$ & $\begin{array}{l}141 \\
61.0\end{array}$ & )$^{24(53.3}$ & $0.3348^{\wedge \wedge}$ & $52(57.8$ & $\begin{array}{l}28(1 \\
62.2)\end{array}$ & )$^{24(53.3}$ & $0.3933^{\wedge \wedge}$ \\
\hline $\begin{array}{l}\text { Concomitant use } \\
\text { of } \\
\text { Aminoglycosides } \\
\text { (Amikacin OR } \\
\text { Gentamicin) for } 2 \\
\text { days or more }\end{array}$ & $\begin{array}{l}114 \\
41.3\end{array}$ & $\begin{array}{l}92( \\
39.8)\end{array}$ & $22(48.9$ & $0.2587^{\wedge \wedge}$ & )$^{40(44.4}$ & $\begin{array}{l}18( \\
40.0)\end{array}$ & $22(48.9$ & $0.3961^{\wedge \wedge}$ \\
\hline
\end{tabular}

\section{Comorbidity}

\begin{tabular}{|c|c|c|c|c|c|c|c|c|}
\hline COPD, n (\%) & $16(5.8)$ & )$^{15(6.5}$ & $1(2.2)$ & $0.2620 * *$ & $3(3.3)$ & $2(4.4)$ & $1(2.2)$ & 0.5571 ** \\
\hline Heart Failure, n (\%) & $16(5.8)$ & )$^{16(7.0}$ & $0(0.0)$ & $0.0683^{* *}$ & $1(1.1)$ & $1(2.3)$ & $0(0.0)$ & $0.3091^{\star \star}$ \\
\hline Hypertension, n (\%) & $\begin{array}{l}113 \\
40.9\end{array}$ & $\begin{array}{l}98( \\
42.4)\end{array}$ & )$^{15(33.3}$ & $0.2565^{\wedge \wedge}$ & $25(27.8$ & $\begin{array}{l}10( \\
22.2)\end{array}$ & )$^{15(33.3}$ & $0.2393^{\wedge \wedge}$ \\
\hline $\begin{array}{l}\text { Diabetes mellitus, } \mathrm{n} \\
(\%)\end{array}$ & $\begin{array}{l}115( \\
41.7)\end{array}$ & $\begin{array}{l}97( \\
42.0)\end{array}$ & )$^{18(40.0}$ & $0.8042^{\wedge \wedge}$ & )$^{33(36.7}$ & $\begin{array}{l}15( \\
33.3)\end{array}$ & )$^{18(40.0}$ & $0.5117^{\wedge \wedge}$ \\
\hline Dyslipidemia, n (\%) & )$^{36(13.0}$ & $\begin{array}{l}28( \\
12.1)\end{array}$ & $8(17.8)$ & $0.3026^{\wedge \wedge}$ & )$^{12(13.3}$ & $4(8.9)$ & $8(17.8)$ & $0.2148^{\wedge \wedge}$ \\
\hline $\begin{array}{l}\text { Hypothyroidism, } \mathrm{n} \\
(\%)\end{array}$ & $18(6.5)$ & )$^{16(7.0}$ & $2(4.4)$ & $0.5332^{\star *}$ & $3(3.3)$ & $1(2.2)$ & $2(4.4)$ & $0.5571 * \star$ \\
\hline $\begin{array}{l}\text { Ischemic heart } \\
\text { disease (IHD), n (\%) }\end{array}$ & $17(6.2)$ & )$^{15(6.5}$ & $2(4.4)$ & $0.5967 * \star$ & $3(3.3)$ & $1(2.2)$ & $2(4.4)$ & $0.5571^{\star \star}$ \\
\hline $\begin{array}{l}\text { Chronic kidney } \\
\text { disease (CKD), n }\end{array}$ & $14(5.1)$ & )$^{12(5.2}$ & $2(4.4)$ & $0.8338^{* *}$ & $4(4.4)$ & $2(4.4)$ & $2(4.4)$ & $>0.9999 * *$ \\
\hline
\end{tabular}

(\%)

\begin{tabular}{|c|c|c|c|c|c|c|c|c|}
\hline $\begin{array}{l}\text { Venous } \\
\text { Thromboembolism, } \\
\mathrm{n}(\%)\end{array}$ & $9(3.3)$ & $9(3.9)$ & $0(0.0)$ & $0.1782^{\star \star}$ & $1(1.1)$ & $1(2.2)$ & $0(0.0)$ & 0.3146 ** \\
\hline $\begin{array}{l}\text { Liver disease (any } \\
\text { type), n (\%) }\end{array}$ & $13(4.7)$ & )$^{13(5.6}$ & $0(0.0)$ & $0.1031^{* *}$ & $0(0.0)$ & $0(0.0)$ & $0(0.0)$ & NA \\
\hline Stroke, n (\%) & )$^{35(12.7}$ & $\begin{array}{l}32( \\
13.9)\end{array}$ & $3(6.7)$ & $0.1851^{\wedge \wedge}$ & $7(7.8)$ & $4(8.9)$ & $3(6.7)$ & $0.6939 * *$ \\
\hline
\end{tabular}

*T Test / ^ Wilcoxon rank sum test is used to calculate the P-value.

${ }^{\wedge \wedge}$ Chi square/ ${ }^{\star \star}$ Fisher's Exact teat is used to calculate P-value.

There were notable differences between the two groups before propensity score matching; patients who received colistinonly were older, had a higher SOFA score, blood urea nitrogen (BUN), International Normalized Ration (INR), and 
mechanically ventilation (MV) needs within 24 hours of ICU admission. Conversely, patients who received colistin concomitant with ascorbic acid therapy have a slightly higher median platelets count and albumin levels baseline.

Following the PS matching (1:1 ratio) based on the selected criteria, 90 patients were included. Most of the baseline characteristics and comorbidities were balanced between the two groups apart from mean body weight, and body mass index (BMI) which was significantly higher in the control group. There was no statistically significant difference between the two groups in concomitant nephrotoxic agent use or exposure (Table 1). The median cumulative dose of IV colistin as CBA in patients who received concomitant ascorbic acid was $68 \mathrm{mg} / \mathrm{kg}$ compared with $56 \mathrm{mg} / \mathrm{kg}$ in the other group. The median ascorbic acid dosage was 1000 mg enterally with a median duration of 36 days.

\section{Outcomes}

\section{Colistin-induced nephrotoxicity (CIN)}

In both groups, older age and higher baseline serum creatinine levels were considered as independent risk factors for CIN. In crude analysis after PS matching, CIN occurred in 16 patients (40\%) who received colistin-only compared to 16 patients (44.4\%) who received colistin concomitant with ascorbic acid therapy $(p=0.69)$. Moreover, at multivariable logistic regression analysis, there was no statistically significant differences between the two groups (OR $0.83 \mathrm{CI} 0.33,2.10 ; p=$ 0.68) (Table 2). The concomitant use of nephrotoxic medications was assessed and not statistically significant before and after PS matching between the two groups (Table 1). 
Table 2

Regression analysis for the outcomes after PS matching

\begin{tabular}{|c|c|c|c|c|c|}
\hline \multirow[t]{2}{*}{ Outcomes } & \multicolumn{2}{|c|}{$\begin{array}{l}\text { Number of outcomes/Total } \\
\text { number of patients }\end{array}$} & \multirow[t]{2}{*}{ P-value } & \multirow[t]{2}{*}{$\begin{array}{l}\text { Odds Ratio (OR) } \\
(95 \% \mathrm{Cl})\end{array}$} & \multirow[t]{2}{*}{$\begin{array}{l}\text { P-value } \\
\$\end{array}$} \\
\hline & $\begin{array}{l}\text { Colistin + } \\
\text { Ascorbic Acid }\end{array}$ & Colistin & & & \\
\hline \multirow{2}{*}{$\begin{array}{l}\text { Colistin-induced nephrotoxicity } \\
(\mathrm{CIN}), \mathrm{n}(\%) \Delta\end{array}$} & $16(44.4)$ & $16(40.0)$ & $0.6952^{\wedge \wedge}$ & $0.83(0.33,2.10)$ & 0.68 \\
\hline & & & & Hazard ratio $(95 \% \mathrm{Cl})$ & $\begin{array}{l}\mathrm{P} \text { - } \\
\text { value\$* }\end{array}$ \\
\hline 30-day mortality, $n(\%) \Delta$ & $8(17.8)$ & $10(22.2)$ & $0.5982^{\wedge \wedge}$ & $1.71(0.65,4.46)$ & 0.27 \\
\hline \multirow[t]{2}{*}{ In-hospital Mortality, $\mathrm{n}(\%) \Delta$} & $12(26.7)$ & $14(31.1)$ & $0.6418^{\wedge \wedge}$ & $1.19(0.55,2.58)$ & 0.66 \\
\hline & & & & $\begin{array}{l}\text { beta coefficient } \\
\text { (Estimates) }(95 \% \mathrm{Cl})\end{array}$ & $\begin{array}{l}\text { P-value } \\
\$ \star{ }^{\star *}\end{array}$ \\
\hline $\begin{array}{l}\text { Hospital LOS, Median (Q1, } \\
\text { Q3) } \Delta\end{array}$ & $\begin{array}{l}56.0(30.00 \\
105.00)\end{array}$ & $\begin{array}{l}52.0(26.00 \\
93.00)\end{array}$ & $0.7165^{\wedge}$ & $0.07(-0.26,0.41)$ & 0.67 \\
\hline ICU LOS, Median (Q1, Q3) $\Delta$ & $\begin{array}{l}35.0(16.00 \\
55.00)\end{array}$ & $\begin{array}{l}20.0(14.00 \\
31.00)\end{array}$ & $0.0281^{\wedge}$ & $-0.05(-0.43,0.33)$ & 0.81 \\
\hline
\end{tabular}

$\Delta$ Denominator of the percentage is the total number of patients.

${ }^{\wedge}$ Chi-square/** Fisher test is used to calculate the P-value.

\$ Multivariate logistic regression analysis is used to calculate Odds ratio and p-value based on patient's age, SOFA score and serum creatinine.

$\$ *$ Cox proportional hazards regression analysis is used to calculate hazard ratio (HR) and p-value.

$\$ \star$ Generalized linear model is used to calculate beta coefficient (estimates) and p-value.

\section{Mortality \& Length of Stay (LOS)}

The 30 -day mortality occurred in 10 patients (22.2\%) who received colistin-only compared to 8 patients (17.8\%) who received colistin concomitant with ascorbic acid therapy $(p=0.5982)$. At multivariable cox proportional hazards regression analyses, the 30-day mortality was higher in patients who received colistin-only; however, it did not reach statistical significance (HR $1.71 \mathrm{Cl} 0.65,4.46 ; p=0.27)$. Moreover, the in-hospital mortality was similar between the two groups (HR $1.19 \mathrm{Cl} 0.55,2.58 ; p=0.66$ ) (Table 2).

Patients who received colistin concomitant with ascorbic acid therapy have a longer ICU stay at crude analysis (35.0 days Vs. 20.0 days, $p=0.02)$. However, there were no statistically significant differences in ICU and hospital LOS between the two groups at regression analysis after using PS matching (Beta coefficient $-0.05 \mathrm{Cl}-0.43,0.33 ; \mathrm{p}=0.81$ and Beta coefficient $0.07 \mathrm{Cl}-0.26,0.41 ; p=0.67$ respectively) (Table 2 )

\section{Discussion}

In our retrospective cohort study of critically ill patients, patients who received colistin with ascorbic acid as adjunctive therapy had similar odds of developing CIN compared to patients who received colistin only. We studied the effect of the low adjunctive dose of ascorbic acid in critically ill patients who received colistin, as it has been demonstrated in preclinical studies that ascorbic acid has a protective effect against the nephrotoxicity and tubular apoptosis caused by colistin. ${ }^{14,16}$ These studies revealed that ascorbic acid could reduce CIN due to its antioxidant properties. ${ }^{14,15}$ The main mechanisms of 
$\mathrm{CIN}$ are acute tubular necrosis, manifested as decreased creatinine clearance. ${ }^{16,17}$ and interstitial nephritis. ${ }^{18}$ However, CIN may be attributable to oxidative damage and inflammation. ${ }^{19}$

In this study, no significant difference was observed in the prevalence of CIN after propensity score matching, which was $35 \%$ in both groups; $p$-value $=0.6952$. These results are consistent with results from a randomized clinical trial conducted on 28 patients; the study did not show any nephroprotection in the ascorbic acid group. ${ }^{11}$ However, the nephroprotective effects of ascorbic acid could not be observed due to the small number of included patients in our analysis and utilization of a lower dose than in other trials (2-4 g of ascorbic acid daily). ${ }^{20,21}$ Higher ascorbic acid dosages or longer administration times may have produced different results. A prospective cohort study of critically ill patients with sepsis. ${ }^{21}$; had comparable results with preclinical studies ${ }^{14,15}$, which showed that ascorbic acid was an independent protective factor against AKI in patients treated with colistin. ${ }^{21}$ The incidence rate of colistin-induced nephrotoxicity was reported in different trials to be in the range between 0 to $53.5 \%,{ }^{6}$ which is consistent with our study results. In addition, the mean age in our study is relatively low $(45.5 \pm 17.74)$, nephrotoxicity has proven to occur more significantly in patients older than 60 years of age. $^{21,22}$

After statistical matching, all patients included in this study had similar baseline kidney function. Two patients (4.4\%) in each group had chronic kidney disease. Similarly, analyzing the effect of nephrotoxic drugs; vancomycin, aminoglycosides, and contrast that were administered during colistin treatment could affect the results. However, after propensity score matching the baseline use of these drugs was not significant between groups. Concomitant use of vancomycin and aminoglycosides for two days or more were $83.3 \%$ and $44.4 \%$, respectively. At the same time, the use of contrast during ICU stay was $57.8 \%$ (Table 1$)$.

Among the 90 patients, we observed that 30-day mortality, in-hospital mortality, and hospital LOS were similar between the two groups. ascorbic acid has been studied in multiple randomized and observational studies in critically ill patients. A meta-analysis study was conducted to evaluate the effects of ascorbic acid administration on clinical outcomes. This metaanalysis included forty-four randomized trials in which 16 of them were performed in an ICU setting. Ascorbic acid administration was not associated with a difference in mortality, acute kidney injury, ICU, or hospital length of stay compared with control. ${ }^{23}$ In addition, the current sepsis guidelines ${ }^{24}$ recommend against ascorbic acid supplementation in critically ill patients. This recommendation was based on an updated analysis that included nine RCTs. Ascorbic acid did not reduce mortality compared to usual care (RR, $0.9 ; 95 \% \mathrm{Cl}, 0.69-1.18)$. Further studies with higher quality may influence future updates by the guidelines. ${ }^{24}$

Our study has the advantage of describing a cohort of critically ill patients treated by colistin with or without ascorbic acid to assess its role in nephroprotection. However, it has several limitations; first, it was a retrospective observational design with a small sample size that may contribute to the analysis and make it unable to detect the differences between groups. Second, it was conducted at a single center, limiting its generability. Third, the possibility of confounder; in our study, the patient's age, SOFA score, and serum creatinine were controlled by statistical matching. Last, the lower dose of ascorbic acid than the doses utilized in other trials might limit reaching a renal protective effect; higher ascorbic acid dosages or longer administration times may have produced different results. Therefore, the results of this study could be used to support the need for further large-scale studies with controlling the confounders to determine whether ascorbic acid has a role in the prevention of colistin-induced nephrotoxicity.

\section{Conclusion}

Concomitant use of ascorbic acid adjunctive therapy with IV colistin in critically ill patients was not associated with lower odds of CIN. Further studies with a larger sample size are required to confirm these findings.

\section{Declarations}

Page $11 / 15$ 


\section{Acknowledgments}

We would like to thank all the investigators who participated in this project from the Saudi critical care pharmacy research (SCAPE) platform.

\section{Author contributions}

All authors made a significant contribution to the work reported, whether that is in the conception, study design, execution, acquisition of data, analysis, and interpretation, or in all these areas; took part in drafting, revising or critically reviewing the article; gave final approval of the version to be published; have agreed on the journal to which the article has been submitted; and agree to be accountable for all aspects of the work.

\section{Funding}

None.

\section{Availability of data and material}

The data that support the findings of this study are available from corresponding author, but restrictions apply to the availability of these data, which were used under license for the current study, and so are not publicly available. Data are however available from the authors upon reasonable request and with permission of corresponding author.

\section{Ethics approval and consent to participate}

The study was approved in March 2021 by King Abdullah International Medical Research Center Institutional Review Board, Riyadh, Saudi Arabia (Ref.\# NRC21R.062.02). Participants' confidentiality was strictly observed throughout the study by using anonymous unique serial number for each subject and restricting data only to the investigators. Informed consent was not required hence it has been waived off from the King Abdullah International Medical Research Center Institutional Review Board, Riyadh, Saudi Arabia due to the retrospective observational nature of the study. All methods were performed in accordance with the relevant guidelines and regulations.

\section{Consent for publication}

Not applicable.

\section{Competing interests}

No author has a conflict of interest in this study.

\section{References}

1. Despotovic, A., Milosevic, B., Milosevic, I., Mitrovic, N., Cirkovic, A., Jovanovic, S., \& Stevanovic, G. (2020). Hospitalacquired infections in the adult intensive care unit-Epidemiology, antimicrobial resistance patterns, and risk factors for acquisition and mortality. American journal of infection control, 48(10), 1211-1215.

https://doi.org/10.1016/j.ajic.2020.01.009 
2. Prestinaci, F., Pezzotti, P., \& Pantosti, A. (2015). Antimicrobial resistance: a global multifaceted phenomenon. Pathogens and global health, 109(7), 309-318. https://doi.org/10.1179/2047773215Y.0000000030

3. Falagas, M. E., \& Kasiakou, S. K. (2005). Colistin: the revival of polymyxins for the management of multidrug-resistant gram-negative bacterial infections. Clinical infectious diseases: an official publication of the Infectious Diseases Society of America, 40(9), 1333-1341. https://doi.org/10.1086/429323

4. Tsuji, B. T., Pogue, J. M., Zavascki, A. P., Paul, M., Daikos, G. L., Forrest, A., Giacobbe, D. R., Viscoli, C., Giamarellou, H., Karaiskos, I., Kaye, D., Mouton, J. W., Tam, V. H., Thamlikitkul, V., Wunderink, R. G., Li, J., Nation, R. L., \& Kaye, K. S. (2019). International Consensus Guidelines for the optimal use of the polymyxins: Endorsed by the American College of Clinical Pharmacy (ACCP), European Society of Clinical Microbiology and Infectious Diseases (ESCMID), infectious diseases society of america (IDSA), International Society for Anti-infective pharmacology (ISAP), Society of Critical Care Medicine (SCCM), and Society of Infectious Diseases Pharmacists (SIDP). Pharmacotherapy: The Journal of Human Pharmacology and Drug Therapy, 39(1), 10-39. https://doi.org/10.1002/phar.2209

5. Gai, Z., Samodelov, S., Kullak-Ublick, G., \& Visentin, M. (2019). Molecular mechanisms of colistin-induced nephrotoxicity. Molecules, 24(3), 653. https://doi.org/10.3390/molecules24030653

6. Spapen, H., Jacobs, R., Van Gorp, V., Troubleyn, J., \& Honoré, P. M. (2011). Renal and neurological side effects of colistin in critically ill patients. Annals of intensive care, 1(1), 14. https://doi.org/10.1186/2110-5820-1-14

7. Temocin, F., Erdinc, S., Tulek, N., Demirelli, M., Bulut, C., \& Ertem, G. (2015). Incidence and risk factors for colistinassociated nephrotoxicity. Japanese Journal of Infectious Diseases, 68(4), 318-320.

https://doi.org/10.7883/yoken.jjid.2014.223

8. Ordooei Javan, A., Shokouhi, S., Sahraei, Z., Salamzadeh, J., \& Azad Armaki, S. (2017). Nephrotoxicity of High and Conventional Dosing Regimens of Colistin: A Randomized Clinical Trial. Iranian journal of pharmaceutical research: IJPR, 16(2), 781-790.

9. Pogue, J. M., Lee, J., Marchaim, D., Yee, V., Zhao, J. J., Chopra, T., Lephart, P., \& Kaye, K. S. (2011). Incidence of and risk factors for colistin-associated nephrotoxicity in a large academic health system. Clinical infectious diseases: an official publication of the Infectious Diseases Society of America, 53(9), 879-884. https://doi.org/10.1093/cid/cir611

10. Dai, C., Li, J., Tang, S., Li, J., \& Xiao, X. (2014). Colistin-induced nephrotoxicity in mice involves the mitochondrial, death receptor, and endoplasmic reticulum pathways. Antimicrobial Agents and Chemotherapy, 58(7), 4075-4085. https://doi.org/10.1128/aac.00070-14

11. Sirijatuphat, R., Limmahakhun, S., Sirivatanauksorn, V., Nation, R. L., Li, J., \& Thamlikitkul, V. (2015). Preliminary clinical study of the effect of ascorbic acid on colistin-associated nephrotoxicity. Antimicrobial Agents and Chemotherapy, 59(6), 3224-3232. https://doi.org/10.1128/aac.00280-15

12. Harish Padh. Cellular functions of ascorbic acid. Biochemistry and Cell Biology. 68(10): 1166-1173. https://doi.org/10.1139/090-173

13. Shrestha, D. B., Budhathoki, P., Sedhai, Y. R., Mandal, S. K., Shikhrakar, S., Karki, S., Baniya, R. K., Kashiouris, M. G., Qiao, X., \& Fowler, A. A. (2021). Vitamin C in Critically III Patients: An Updated Systematic Review and Meta-Analysis. Nutrients, 13(10), 3564. https://doi.org/10.3390/nu13103564

14. Yousef, J. M., Chen, G., Hill, P. A., Nation, R. L., \& Li, J. (2011). Ascorbic acid protects against the nephrotoxicity and apoptosis caused by colistin and affects its pharmacokinetics. Journal of Antimicrobial Chemotherapy, 67(2), 452459. https://doi.org/10.1093/jac/dkr483

15. Aslan, T., Guler, E. M., Cakir, A., Dundar, T., Gulgec, A. S., Huseyinbas, O., ... Durdu, B. (2021). Dexpanthenol and ascorbic acid ameliorate colistin-induced nephrotoxicity in rats. European Review for Medical and Pharmacological Sciences, 25(2), 1016-1023.

16. Zavascki, A. P., \& Nation, R. L. (2017). Nephrotoxicity of polymyxins: is there any difference between colistimethate and polymyxin B?. Antimicrobial agents and chemotherapy, 61(3), e02319-16. 
17. Javan, A. O., Shokouhi, S., \& Sahraei, Z. (2015). A review on colistin nephrotoxicity. European journal of clinical pharmacology, 71(7), 801-810.

18. Kallel, H., Hamida, C. B., Ksibi, H., Bahloul, M., Hergafi, L., Chaari, A., ... Bouaziz, M. (2005). Suspected acute interstitial nephritis induced by colistin. Journal of nephrology, 18(3), 323-326.

19. Ozyilmaz, E., Ebinc, F. A., Derici, U., Gulbahar, O., Goktas, G., Elmas, C., ... Sindel, S. (2011). Could nephrotoxicity due to colistin be ameliorated with the use of $\mathrm{N}$-acetylcysteine?. Intensive care medicine, 37(1), 141-146.

20. Kellum, J. A., Lameire, N., Aspelin, P., Barsoum, R. S., Burdmann, E. A., Goldstein, S. L., ... Uchino, S. (2012). Kidney disease: improving global outcomes (KDIGO) acute kidney injury work group. KDIGO clinical practice guideline for acute kidney injury. Kidney international supplements, 2(1), 1-138.

21. Dalfino, L., Puntillo, F., Ondok, M. J. M., Mosca, A., Monno, R., Coppolecchia, S., ... Brienza, N. (2015). Colistin-associated acute kidney injury in severely ill patients: a step toward a better renal care? A prospective cohort study. Clinical Infectious Diseases, 61(12), 1771-1777.

22. Balkan, I. I., Dogan, M., Durdu, B., Batirel, A., Hakyemez, I. N., Cetin, B., ... Tabak, F. (2014). Colistin nephrotoxicity increases with age. Scandinavian journal of infectious diseases, 46(10), 678-685.

23. Putzu, A., Daems, A. M., Lopez-Delgado, J. C., Giordano, V. F., \& Landoni, G. (2019). The effect of vitamin C on clinical outcome in critically ill patients: a systematic review with meta-analysis of randomized controlled trials. Critical care medicine, 47(6), 774-783.

24. Evans, L., Rhodes, A., Alhazzani, W., Antonelli, M., Coopersmith, C. M., French, C., ... Levy, M. (2021). Surviving sepsis campaign: international guidelines for management of sepsis and septic shock 2021. Intensive care medicine, 47(11), $1181-1247$.

\section{Figures}




\section{Inclusion:}

Adult patients (Age $\geq 18 \mathrm{y} / \mathrm{o}$ ) who were using IV Colistin in the ICU between 01/01/2017 and $31 / 12 / 2020$.

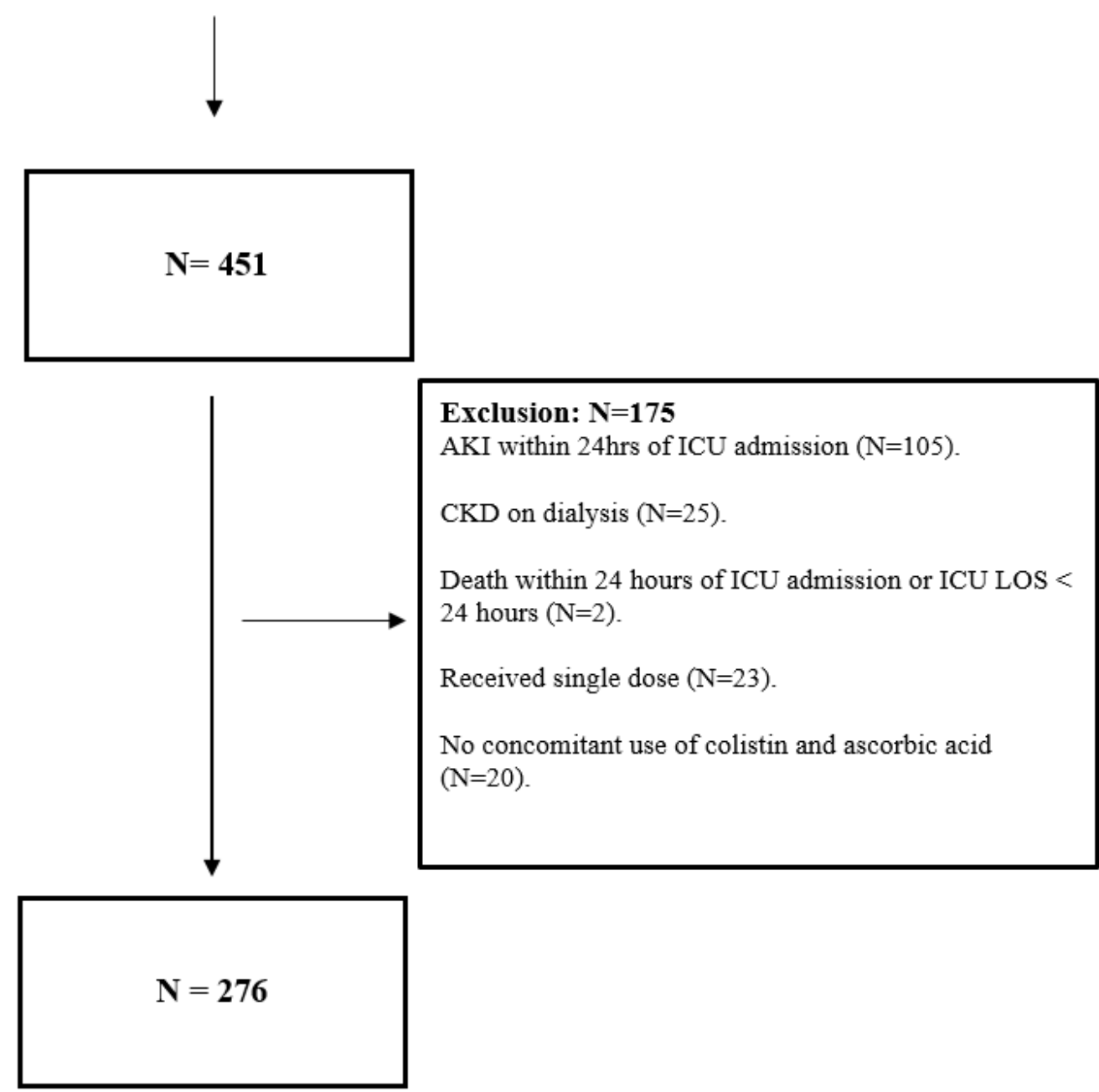

Figure 1

Flow diagram of inclusion/exclusion criteria, and for eligible patients who underwent analysis. 